\title{
Beam cooling: principles and achievements
}

\author{
Dieter Möhl, ${ }^{\mathrm{a}, 1}$ Andrew M. Sessler, ${ }^{\mathrm{b}, 2}$ \\ ${ }^{a}$ CERN, CH-1211 Genève 23, Switzerland \\ ${ }^{b}$ Lawrence Berkeley Laboratory, 1 Cyclotron Road, Berkeley, CA 94720, USA
}

Elsevier use only: Received date here; revised date here; accepted date here

\begin{abstract}
After a discussion of Liouville's theorem, and its implications for beam cooling, a brief description is given of each of the various methods of beam cooling: stochastic, electron, radiation, laser, ionization, etc. For each, we present the type of particle for which it is appropriate, its range of applicability, and the currently achieved degree of cooling. For each method we also discuss the present applications and, also, possible future developments and further applications.
\end{abstract}

29.27 Beams in particle accelerators, 41.75.-i Charged-particle beams, 41.85.-p Beam optics

Keywords: beam cooling, Liouville, stochastic, electron, laser, cooling methods

\section{Introduction and Liouville's Theorem}

Fifty years ago there were no cooling methods for hadrons and the idea of "radiation damping" of electron beams was just starting to emerge. Yet, in the mid-50s, the MURA Group was aware of the desirability of phase space compression for protons, but was unable to think of any such cooling method. Now-a-days, cooling is essential to many particle storage rings and its importance is ever-growing. Thus within our professional lifetime we have experienced a revolution.

In this introductory paper we want to briefly outline the various cooling methods and, also, present current achievements and future possibilities. There are many reasons for cooling beams, and we present them, categorized, in Table 1.

Cooling is done in special cooling rings and also in some very large machines such as synchrotron radiation facilities. The cooling rings supply very dense beam either for direct use in precision experiments or for injection into big, high luminosity machines like the Tevatron or the projected next Linear Colliders, or the proposed Neutrino Factories. In Table 2 we list the special cooling rings used for precision experiments.

To appreciate these achievements, and to distinguish what is phase space cooling and what is not, it is appropriate to think deeply about the Liouville Theorem.

\footnotetext{
${ }^{1}$ Dieter Möhl Tel: + 41-22-767-30-35-611; e-mail: Dieter.Mohl@cern.ch

2 Andrew Sessler Tel.: +1-510-486-4992; fax: +1510-486-6485; e-mail: AMSessler@lbl.gov.
} 


\section{Table 1 Reasons for Cooling Beams}

1. For colliding proton -antiproton beams such as the SPS, or the Tevatron, or the GSI-project

Stochastic cooling

(for the accumulation of antiprotons)

Electron cooling

(for the "recycling" and "conditioning" of

antiprotons)

2. For colliding electron-positron beams such as in LEP, Tristan, Future Linear Colliders

Radiation Cooling

3. For gamma-gamma colliders

Radiation cooling

Laser Radiation cooling

4. For high energy ion colliders such as RHIC, LHCION-Programme

Electron cooling

(for accumulation of ions at low energy)

Stochastic or electron cooling

(to counteract luminosity decay at collision

energy)

5. For crystal beam studies

Electron cooling

Laser cooling

6. For future radioactive ion colliders (MUSES,

GSI...)

Stochastic and electron cooling

(for accumulation of rare ions)

7. For nuclear and atomic physics (all cooler rings), low and medium energy antiproton physics

(LEAR/AD, GSI project)

Electron and stochastic cooling

(to increase resolution, to counteract scattering

blow- up on internal targets)

8. For neutrino factories and muon colliders Ionization cooling

9. For synchrotron radiation sources such as APS, ESR, ALS, Spring 8

Radiation cooling
Table 2 Cooler rings for Precision Experiments

At Present:

\author{
AD-Geneva (CERN) \\ ASTRID Aarhus \\ ELISA - Aarhus \\ CELSIUS- Uppsala \\ COSY- Juelich \\ CRYRING-Stockholm \\ ESR-Darmstadt (GSI) \\ FERMILAB p-bar source \\ IUCL-Indiana \\ TSR -Heidelberg \\ E-RING - KEK
}

In the future:

ACR,NESR,HESR- Darmstadt (GSI)

IMP-Lanzhou (China)

KEK-JAERI antiproton accumulator

LEPTA - Dubna (JINR) positron ring

LEIR - Geneva (CERN) ion accumulator

MUSES - Tokyo (RIKEN)

S-LSR - Kyoto

Cooling "experiments":

HIMAC - (Shiba, Japan)

PALLAS-Munich

\subsection{The Actual Liouville Theorem}

This dynamical theorem [1] applies in a conservative Hamiltonian system such as a single particle in external magnetic and electric fields. We can define the phase density of many non-interacting particles by considering systems each of which has slightly different initial conditions. The theorem states that phase density is preserved as one follows the motion of the particles.

Thus, this theorem is applicable to a beam when the interaction between particles can be ignored, which is often true-but not always true-for high energy beams.

Even within the restrictions of Liouville's theorem it is possible to arrange to interchange phase space (between, say, longitudinal and transverse degrees of freedom), or equally interestingly, to introduce correlations between the degrees of freedom, i.e., "push phase space around". 


\subsection{Liouville's Theorem for a Continuous Medium}

The study of the motion of particles in an accelerator becomes a many-body problem when the interactions between particles are taken into account. It is thus important to investigate the possibility of establishing the validity-or approximate validity - of general dynamical theorems applicable to the n-body problem. Such a powerful theorem is Liouville's theorem for a dense medium with conservative interactions. We call this the Generalized Liouville Theorem. This is an extremely good approximate theorem for particles in an accelerator.

The Liouville theorem asserts that in a $2 \mathrm{fN}$ dimensional space ( $f$ is the number of degrees of freedom of one particle), spanned by the coordinates and momenta of all particles (called $\gamma$ space), the density in phase is a constant as one moves along with any phase point. It is thus a statement about the density of points, each point representing a dynamical system. The systems constitute an ensemble and of course do not interact.

The Continuous Medium Theorem refers to a system of infinitely many interacting particles; i.e., a continuous medium. It asserts that in the $2 \mathrm{f}$ dimensional space spanned by a single system of coordinates and momenta (called a $\mu$ space), the density in phase is a constant as one moves along with any phase point. It is thus a statement about the behavior of interacting particles, and thus really quite different from the original Liouville's theorem. It has been proved rigorously in [2].

\subsection{Intuitive Proof of the Generalized Liouville Theorem}

The validity of the Generalized Theorem, as well as the limits of its validity, may readily be seen by the following intuitive argument:

Consider first a system of many particles, $N$. Suppose these particles are subject to external forces (which may even be time-dependent), but there are no interactions between the particles. Clearly density in phase in $\mathrm{m}$ space is a constant of the motion as one follows the motion of a phase point. This follows then immediately from Liouville's Theorem in $\gamma$ space, since with no interactions between particles, $\mathrm{m}$ space for $N$ particles, is simply $\gamma$ space for a single particle.

Consider now a system of a great many particles $N$, with interactions between the particles. Imagine that the solution has been obtained so that we know the motion of all the particles as a function of time.
Concentrate now on a "small" number of particles $n$, which initially are localized in $\mu$ space. We will define what "small" means shortly. Let all the other particles move along the trajectories appropriate to the solution of the $N$-body problem. If the interactions between one of the particles and the $n$ particles can be neglected compared to the interactions between the $N-n$ particles and one particle, then these particles are subject to "external forces" and by the first case the density in $\mu$ space is a constant as one moves along with the sample group of $\mathrm{n}$ particles. This is clearly true for any sample, and hence the theorem is established.

That is, as long as one has sufficient particles $N$, that a sample can be obtained of sufficiently small number of particles $n$, that the interactions between one of these $n$ particles and the rest of the $n$ particles is negligible compared to the interactions between one of these particles and the $N-n$ particles, while at the same time $\mathrm{n}$ is sufficiently large that fluctuation phenomena can be neglected, then the theorem is valid.

\subsection{Cooling}

Clearly each of the cooling methods must "get around the theorem"; that is, in one way or another not satisfy the assumptions. Each method does exactly that.

\section{Stochastic Cooling}

The name "stochastic damping" was coined by van der Meer, who invented the concept, to underline the statistical basis of the method [3]. At its core is the observation, that the phase-space density can be increased by a system of pick-up and kicker similar to the arrangement used to damp coherent beam oscillations. The stochastic damping system acts to reduce the deviation of small circumferential sections, called samples, of the beam. By measuring and correcting the statistical fluctuations (baptised "Schottky noise") of the sample averages, the spreads in the corresponding beam properties are gradually reduced. Stochastic cooling may thus be viewed as a "sampling procedure" where samples are continuously taken from the beam and the average of each sample is corrected. At each passage, a particle in the sample receives its own "coherent" kick plus the "incoherent" random kicks due to all other sample members. The coherent effect depends linear and the incoherent effect quadratically on the amplification between pick up and kicker and their 
balance determines the range of amplifier gain where the cooling predominates. An overview of a stochastic cooling system is shown in Fig 1.

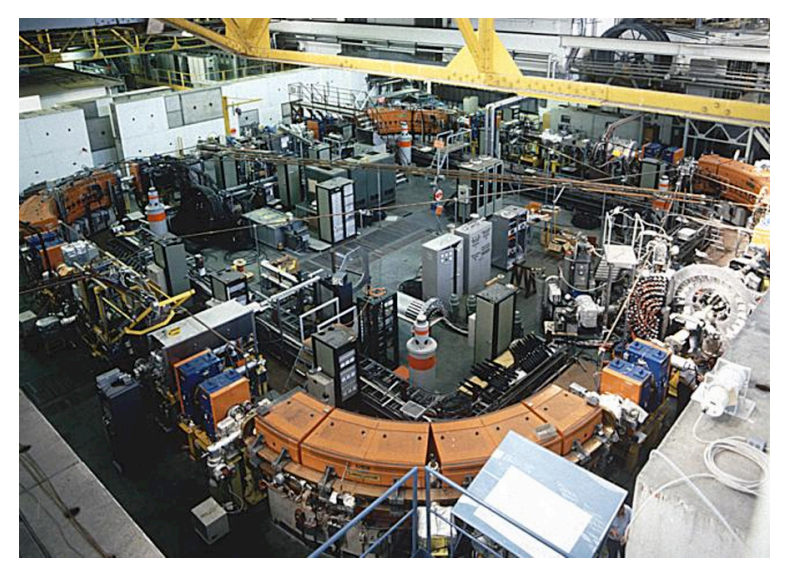

Fig 1. Stochastic Cooling at LEAR (CERN). Different signal transmission lines cutting through the ring can be seen (CERN photo 9007366)

The sample length is given by response-time of the system which in turn is determined by the bandwidth and, hence, large bandwidth is important because small samples show large fluctuations and produce less incoherent kicks on the test particle.

We note in passing that it is exactly for the reason of large bandwidth that the concept of optical stochastic cooling (using optical frequency bands) has been proposed by Zolotorev and Zholents [4]. The principle is no different than in regular stochastic cooling, but the technology is completely different. Wigglers replace the pick ups and kickers, and laser media amplifiers replace electronic amplifiers.

The well-known "mixing dilemma" arises because stochastic cooling only works if after each correction, the samples (at least partly) re-randomize (desired mixing), and at the same time a particle on its way from pick-up to kicker does not slip too much with respect to its own signal (undesired mixing). Proper lattice design and proper placement of pick-up and are important issues. The final performance is always the result of a compromise between desired and undesired mixing.

It is vital to understand, and reduce, system noise. Electronic fluctuations on the low-level side act in a similar way as the particles of the sample on a test particle. The influence can be very critical, given the small signals and the required high amplification. The noise to signal ratio depends on the technology of the pre-amplifier and other "low level components" on the one hand and on the sensitivity of the pick-up device on the other hand. There has been great progress in the design of the pick-up and kicker structures. At the same time the other components in the cooling loop have been steadily improved. Modern stochastic cooling systems combine in unprecedented ways, equipment of high sensitivity, low noise, great bandwidth, large amplification, and very linear phase response. All this is accomplished with compatibility with the ultra-high vacuum of the storage ring.

\section{Electron Cooling}

The idea of cooling was introduced by Budker and Skrinsky [5]. The notions of "beam temperature" and "beam cooling" were introduced to characterize this process, which is readily viewed as temperature relaxation in the mixture of a hot ion beam with a comoving cold electron "fluid". The equilibrium emittances, obtainable when other "heating mechanisms" are negligible, can easily be estimated from this analogy, assuming equalization of the temperatures. For a simple estimate of the cooling time, another resemblance, namely the analogy with slowing down of swift particles in matter, can be helpful.

The cooling rate exhibits dependence on beam properties and storage ring parameters. Notable is the dependence on both the electron and the ion velocity spreads. In the "ion spread dominated regime", cooling gets faster as the ions cool down until it saturates in the "electron dominated regime". Remarkable also is the strong energy dependence, $\tau \sim \beta^{4} \gamma^{5}$, predicted in this model.

Neglected in the simple theory are the "flattened distribution", the "magnetization" and the "electron space-charge" effects. In essence the flattened distribution effect takes into account that (due to the acceleration) the electron velocity spread is not isotropic but contracted in the longitudinal direction. The magnetization effect is due to the spiraling (Larmor) motion of the electrons in the magnetic field of the solenoid that is used to guide the electron beam. The electron space-charge effect is due to the electron's space charge inducing a potential that leads to a parabolic velocity profile $\mathrm{v}(\mathrm{r})$ over the beam whereas the ions exhibit a linear dependence $\mathrm{v}(\mathrm{x})$ given by the storage ring lattice. Hence the difficulty to match the ion and electron velocities. Flattening and magnetization favor cooling because the electrons look colder (less effective velocity spreads) whereas the space-charge effect is hampering. 
Cooling Rings, employing this technique (as well, typically, as stochastic cooling) are listed in Table 2. A typical electron cooling system is shown in Fig 2.

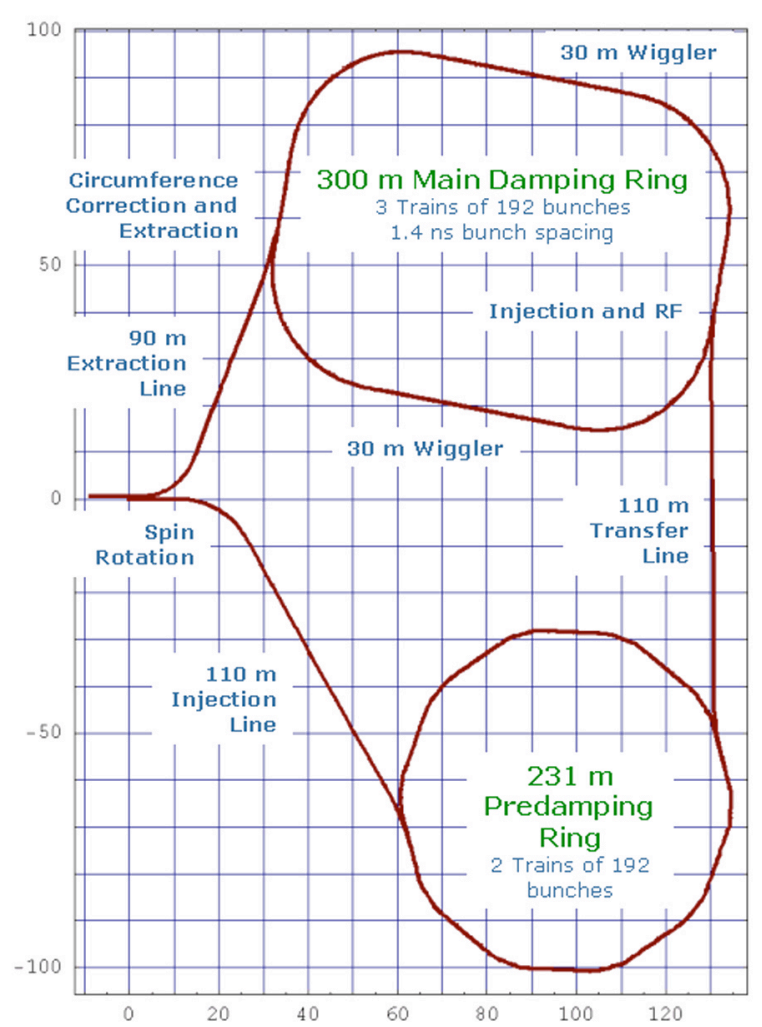

In 1956, A.A. Kolomenski and A.N. Lebedev [6] pointed out that the synchrotron light emitted by an electron on a curved orbit can have a damping effect on the motion of the particle. This was quantified and made specific by Robinson [7]. Because the radiation is sharply peaked in the forward direction, the continuous emission of synchrotron radiation leads to a frictional force opposite to the direction of the motion. The result is both energy loss i.e., deceleration and reduction of the normalized emittances i.e., cooling. The average energy loss is restored by the rf-system.

The cooling force is counteracted by the "radiation excitation": synchrotron light is really emitted in discrete quanta and these many small kicks tend to heat the particle. The final emittances result from the equilibrium of radiation damping and excitation.

The theory is in a mature state based upon Sands' classical treatment [8] on the physics of electron storage rings. The immense success of modern electron-positron machines, both "synchrotron light facilities" and colliders would not have been possible without the full understanding of radiation effects.
Linear $\mathrm{e}^{+} \mathrm{e}^{-}$-collider schemes rely on "damping rings" in their injector chain to produce the ultra-high phase-space density required. The proposed damping rings for the NLC and for TESLA are shown in Figs 3 and 4.

Fig 3. The proposed layout for the NLC (radiation) damping rings. (Taken from the NLC Design Report)

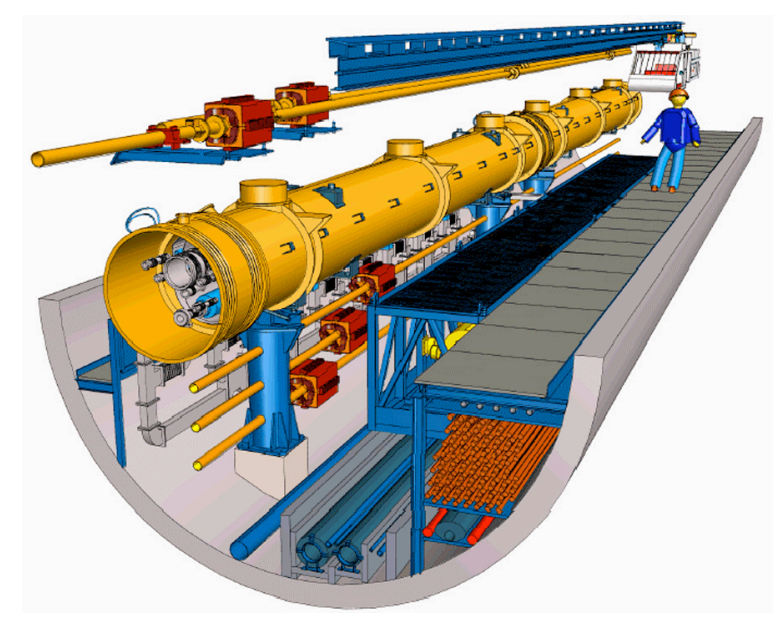

Fig 4. An artist's view of the TESLA damping ring. The damping ring is suspended from the ceiling (the main linear accelerator is below).(Taken from the TESLA Design Report) 
The cooling rates as well as the final beam size and momentum spread depend on the lattice. The art is then to "arrange" these functions such that the desired beam property results. By smart design, gains of orders of magnitude in the equilibrium emittances have been obtained.

\section{Laser Radiation Cooling}

Laser cooling of electrons was first mentioned in a talk by R. Palmer, but then studied in detail by V. Telnov [9]. The idea is essentially to force the electrons to radiate by subjecting them to the very strong field of a laser (rather than a static magnet in a circular machine). When applied to electrons in (say) a linear collider (of several $\mathrm{GeV}$ ), the laser forces the radiation of low energy photons (a few eV). These photons are essentially along the electrons direction and neither the transverse size of the beam or the angles in the beam are altered. However the energy is reduced by the process, and thus the normalized emittance is reduced.

The process is exactly the same as in normal radiation damping (Section IV) and similar to that in ionization cooling (Section VII) and that in frictional cooling (Section VIII), except that the friction force here is due to the stimulated emission.

The electrons may be accelerated to their original energy, $\mathrm{E}_{0}$, and the process repeated $\mathrm{N}$ times. The normalized emittance will be reduced by the amount $\left(E_{1} / E_{0}\right)^{N}$, where $E_{1}$ is the energy of the electrons after interaction with the laser.

There are many considerations and, also, many complications, such as the increase in energy spread of the beam and the reduction of beam polarization due to the process, both, unfortunately, very important for linear collider applications. Perhaps the most serious problem is the need for a very powerful laser capable of both high peak and high average power.

\section{Laser Cooling of Circulating Ion Beams}

Due to the pioneering work of the Heidelberg (TSR) [10] and Aarhus (ASTRID) [11] groups during the last decade, laser cooling in storage rings has rapidly evolved into a very powerful technique. Longitudinal cooling times as short as a few milliseconds and momentum spreads as small as $10^{-6}$ have been achieved. However, laser cooling takes place (mainly) in the longitudinal plane and it works (only) for special ions that have a closed transition between a stable (or meta-stable) lower state and a short-lived higher state.

The principle is, by now, well-known: A transition is excited by laser light, and velocity dependant "absorption" of the light can be arranged to lead to longitudinal cooling. The return to the lower state occurs through spontaneous re-emission and leads to heating. The un-closed transitions, where the deexcitation to more than one level is possible, are not suited because ions decaying to the "wrong" states are lost for further cooling cycles. Up to now, only a few singly charged ions (like $\mathrm{Li}^{1+}, \mathrm{Be}^{1+}$ or $\mathrm{Mg}^{1+}$ ) have been used.

Through transverse-to-longitudinal coupling, part of the cooling can be transferred to the horizontal and vertical planes. Intra-beam scattering, dispersion and special coupling cavities have been considered for this purpose.

The main motivation for laser cooling has been the goal of achieving ultra-cold crystalline beams where the ions are held in place because the Coulomb repulsion overrides the energy of their thermal



motion. A diagram of a laser cooling facility is shown in Fig 5.

Fig 5. Layout of the ASTRID storage ring where two lasers are used for cooling and an additional laser for beam diagnostics (curtesey of N. Madsen, Aarhus and Zurich university)

Laser cooling in storage rings has lead to very interesting and important results concerning the physics of cooling and cooling rings and, also, some fine atomic and laser physics, however, "accelerator applications", such as for electron or stochastic cooling, have not yet been achieved. 


\section{Ionization Cooling of Muons}

The need to cool muons quickly (the muon lifetime is in its frame $2.2 \mu \mathrm{s}$ ) requires a method different from those used previously. Ionization cooling, first suggested by Budker and Skrinsky [12] is employed and it is quite analogous to radiation cooling only in this case energy is lost in materials by ionization, rather than by radiation of an accelerated charge. To that end an absorber is placed into the beam and after the absorber the beam energy is restored by rf-acceleration. The energy loss in the absorber leads to a reduction in normalized emittance, but it is balanced by multiple scattering. The basic equations is:

$$
\begin{gathered}
\frac{d \varepsilon_{n}}{d s}=-\frac{1}{\beta^{2}}\left|\frac{d E_{\mu}}{d s}\right| \frac{\varepsilon_{N}}{E_{\mu}}+\frac{\beta_{\perp}(0.014 G e V)^{2}}{2 \beta^{3} E_{\mu} m_{\mu} X_{o}} \\
\text { cooling heating }
\end{gathered}
$$

The preference is for strong transverse focussing (low $\beta_{\perp}$ ), and large energy loss $(\mathrm{dE} \mu / \mathrm{ds})$ and large scattering length $\left(\mathrm{X}_{0}\right)$. Thus the choice of absorber material is important, with hydrogen the best. Choice of muon energy is dictated by a balance between scattering and longitudinal emittance growth; 200 $\mathrm{MeV}$ seems like a good compromise.

Longitudinal cooling is accomplished by transfering phase space between transverse and longitudinal directions by a wedge shaped absorber. As is the case for radiation damping, the sum of the three cooling decrements is constant and hence stronger longitudinal cooling reduces the transverse cooling speed. A great deal of work has recently been done on cooling rings. These are proving very effective as they can be made to cool in both longitudinal and transverse directions. At the same time, the cooling components are re-used (perhaps 10 , or so, circuits are made by the muons), so the cost of the cooling section is reduced.

An international cooling experiment has been proposed and is shown in Figs. 6 and 7.

\section{Frictional Cooling of Muons}

The basic idea of frictional cooling is to bring the muons into a kinetic energy range where the energy loss increases with kinetic energy [13]. This is either very high energy or energy in the range of a few $\mathrm{keV}$ which is the range employed in frictional cooling. At low energy a muon does not ionize atoms, but rather losses energy by excitation of the gas atoms, elastic scattering on nuclei and charge exchange reactions yielding differences for $\mu^{+}$and $\mu^{+}$. In principle the method works for both positive and negative muons.

The crucial question, for application of the cooling method to a Neutrino Factory, is the efficiency of the scheme. Existing schemes miss the required efficiency by many orders of magnitude. The Columbia University Group has developed a scheme that shows some promise of reaching the desired efficiency and phase space goal, but there are many questions still to be answered. These include muonium formation for postive muons (and hence the use of helium for the stopping gas), and muon capture for negative muons (and hence the use of a low-Z gas and high (but still below the peak in the ionization curve) kinetic energy for the muons).

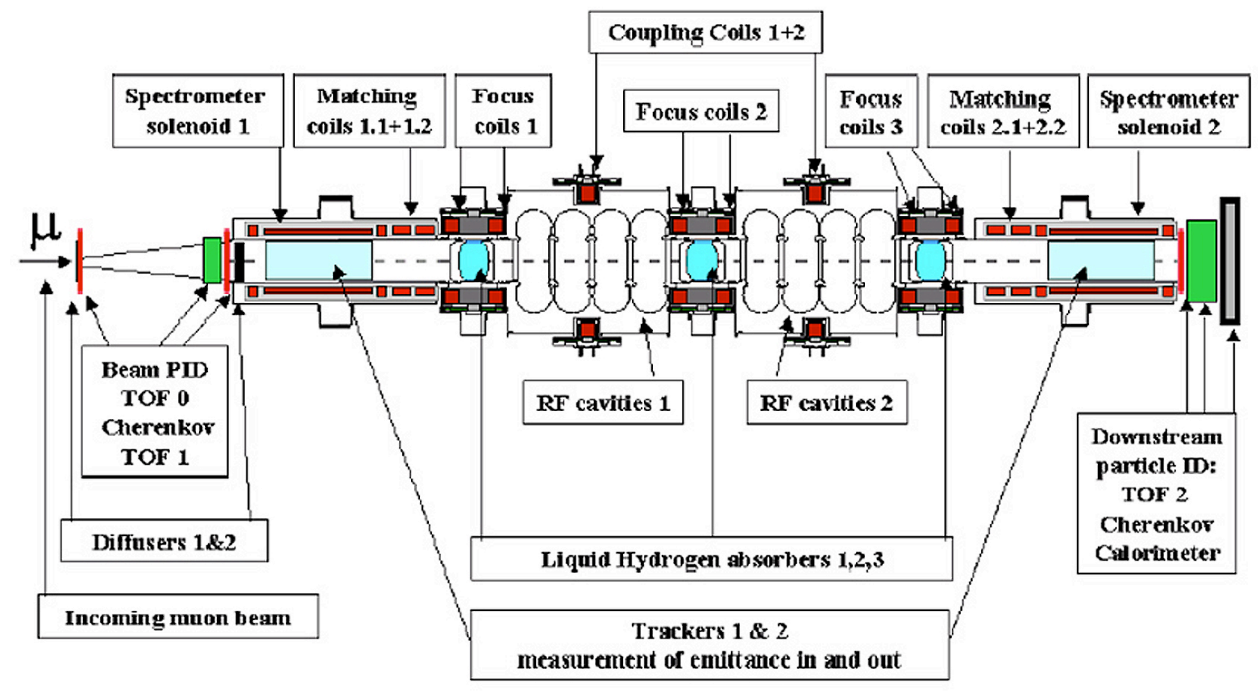

Fig. 6. The proposed Muon Ionization Cooling Experiment MICE (taken from the MICE Proposal) 


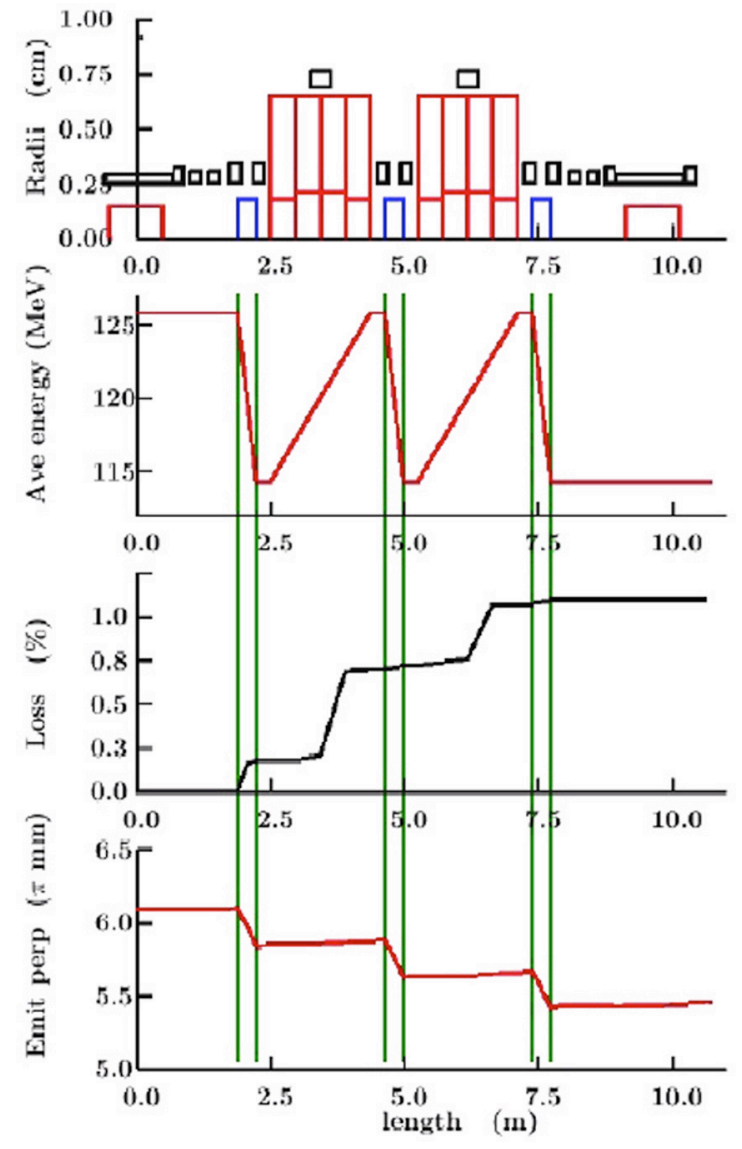

Fig 7. Simulation with the code ICOOL of the MICE experiment showing transverse emittance reduction of ${ }^{\mathrm{a}} 10 \%$. Energy variation, particle loss and emittance reduction are displayed. (Taken from the MICE Proposal)

The proposed scheme is a series of solenoids (providing a longitudinal magnetic field) with gas (cooling) cells between them each having a transverse electric field. The muons travel longitudinally, but as they become cooled they exit transversely. The motion is essentially that of a collapsing helical trajectory; that is, as the muons lose energy the magnetic field becomes less effective and, finally, under the influence of the electric field, the muon exits transversely. Capturing and combining the streams of muons, without degradation of emittance, is a major challenge. Experimental work is underway at Columbia and, by the same team, at the Max-Planck-Institut fuer Physik in Munich [13].

\section{Stimulated Laser Cooling}

It has been observed by Bessonov and Kim [14] that a non-fully-ionized ion beam can be stimulated (by a properly tuned laser) so as to cause it to radiate significantly. Thus, one can achieve radiation damping in circumstances where natural radiation damping is negligible (due to the ion's large mass). Although a laser is employed, this is very different from laser cooling as described in Section VI. In particular, and in marked contrast with the usual laser cooling method, but as in radiation cooling, damping of emittance by a factor of $\mathrm{N}$, requires that the ion lose an amount of energy equal to $\mathrm{N}$ times its energy.

The scheme involves excitation of a bound electron to another bound state by a laser that is tuned to be very close to the transition frequency. On resonance the cross section for excitation is (typically) 18 orders of magnitude larger than off resonance. But, of course, the laser can't be on resonance for all ions due to the temperature of the beam and the resulting Doppler shifts. Because of the many orders of magnitude to burn, the laser can be broad band enough to excite all of the ions and the average cross section is still very interesting for cooling. The ion absorbs a photon and then spontaneously radiates symmetrically. Thus the transverse momentum damps to zero. The scheme also works longitudinally because the faster particles lose more kinetic energy than the slower ones. Detailed rates of damping are evaluated in Ref. 14.

\section{Conclusion}

Much has been achieved with beam cooling, including:

1. All discoveries made with $\mathrm{e}^{+} \mathrm{e}^{-}$machines since 1960s (including charm, tau, synchrotron light physics) with radiation cooling,

2. W, Z, top, anti-H with stochastic cooling,

3. Beams of unprecedented brightness by electron cooling,

4. Special ion beams of still higher brightness by laser cooling,

5. A wide field of fascinating accelerator physics.

The different cooling methods have opened fields of physics not otherwise possible to be addressed. Further, the "old" methods are far from exhausted and a number of "new" cooling methods appear to be on the horizon. Perhaps it is appropriate to end with 
a statement of the fantastic, so-far-accomplished, cooling achievements:

Intensity increase:

1. Antiprotons : factor: $>10^{5}$ ( FERMILAB and CERN AA)

2. Ions : factor: $>10^{2}$ (in the future maybe $10^{3}$ $\left.-10^{5}\right)$

Increase of 6D phase space density:

1. In $\mathrm{AA}:>10^{9}$

2. In $\mathrm{AD}:>10^{11}$

In summary we can say, beam cooling has led, leads, and will lead to spectacular results. In addition beam cooling was, is, and will be fun! What more do you want?

\section{References}

[1] J. Liouville., Journ. De Math. 3, 349 (1838).

[2] R.L. Mills and A.M. Sessler, "Liouville's Theorem and Phase Space Cooling", Montreux Proceedings, CERN Report 9403, 4 (1994).
[3] S. van der Meer., "Stochastic Damping of Betatron Oscillations...," internal report CERN ISR-PO 72/31 (1972).

[4] M. Zolotorev and S. Zholents, Phys. Rev. E 50, 3087 (194).

[5] G.I.Budker, A.N. Skrinsky, Sov. Phys. Usp. 124, 277 (1978).

[6] A.A. Kolomenski, A.N. Lebedev, "The Effect of Radiation on the Motion of Relativistic Electrons in Synchrotrons", in: Proc. CERN Symposium, p. 447 (1956).

[7] K.W. Robinson, Phys. Rev. 111, 373 (1958)

[8] M. Sands, The Physics of Electron Storage Rings ..., in: Proc. Internat. School 'Enrico Fermi', $46^{\text {th }}$ course, Varenna 1969, (ed. B. Touschek,) Academic Press, NY 1971 (preprint: SLAC report 121, 1970).

[9] V. Telnov, Phys. Rev. Lett. 78, 4757 (1997), erratum ibid 80, 2747 (1998); Nucl. Instr. and Meth. A450, 63 (2000).

[10] S. Schroder et al., Phys. Rev. Lett. 64, 2901 (1990); W. Petrich et al., Laser Cooling at the Heidelberg Test Storage Ring (TSR), in: Proc. Workshop on Electron Cooling ..., Legnaro (1990), World Sci. Publishing Company, Singapore (1991) ( R. Calabrese and L. Tecchio, eds.) p. 243.

[11] J. Hangst et al., Phys. Rev. Lett. 67, 1238 (19891); J.S. Hangst et al, Nucl Instrum. Meth. A441, 196 (2000).

[12] G.I. Budker, in: Proc. of $15^{\text {th }}$ Internat. Conf. on High Energy Phys., Kiev 1970 and A.N. Skrinsky, Intersecting Storage Rings at Novosibirsk, in: Proc. of Morges Seminar 1971, Report CERN/D.PH II/YGC/mng. A more recent review is givn by A.N. Skrinsky, Nucl. Instrum. Meth. 391, 188 (1997).

[13] M. Muhlbauer, et al, Hyperfine Interact. 119, 305 (1999); A. Caldwell, "Conceptual Design for a Frictional Cooling Scheme", Rinton and private communication.

[14] E.G. Bessonov and K-J Kim, Phys. Rev. Lett. 76, 431 (1996). 